\title{
Genetically-Defined DNA Repair Capacity Determines the Extent of DNA Damage Accumulation in Healthy Mouse Tissues after Very Low Doses of Ionizing Radiation
}

\author{
Stefanie Schanz ${ }^{\#}$ Elias Flockerzi ${ }^{\#}$, Karola Schuberth, Claudia E. Rübe \\ Department of Radiation Oncology, Saarland University, Homburg/Saar, Germany \\ "Corresponding author: Claudia E. Rübe, Department of Radiation Oncology, Saarland University, Kirrbergerstr. Geb.6.5, 66421 Homburg/Saar, Germany, Tel: +49 6841 \\ 1634614; Fax: +49 6841 1624699; E-mail: claudia.ruebe@uks.eu \\ \#These authors contributed equally to this manuscript.
}

Rec date: Sep 26, 2014, Acc date: Oct 30, 2014, Pub date: Oct 31, 2014

Copyright: () 2014 Schanz S, et al. This is an open-access article distributed under the terms of the Creative Commons Attribution License, which permits unrestricted use, distribution, and reproduction in any medium, provided the original author and source are credited.

\begin{abstract}
The biological impact of low doses of ionizing radiation on human health and the genetic factors influencing whole organism radio-sensitivity at low doses are unclear. Using mouse strains that varied in genetic DNA repair capacity (C57BL/6, ATM +/+, ATM +/-, ATM -/-, SCID), we analyzed DNA damage in differentiated cell populations of healthy tissues after repeated low doses of radiation. After $2,4,6,8$, and 10 weeks of daily, low-dose radiation (10 mGy), persistent DNA damage foci were counted in the lung (bronchiolar and alveolar cells), heart (cardiomyocytes), and brain (cortical neurons). In all analyzed tissues, the gradual accumulation of DNA damage with increasing doses of fractionated radiation was observed. No verifiable threshold-dose was detected, even in repair-proficient organisms (C57BL/6, ATM +/+). The number of radiation-induced foci varied significantly between the different cell populations, suggesting differing vulnerability to ionizing radiation. Genetic DNA repair capacity also determined the cumulative amount of low-dose radiation damage, with the highest foci levels observed in repair-deficient ATM -/- and SCID mice. The repair capacity of ATM heterozygous mice (ATM +/-), however, was sufficient to cope with the DNA damage burden induced by repetitive low-dose radiation. Collectively, our findings suggest that even very low doses of DNA-damaging radiation increase the health risks of individuals, particularly of those with compromised DNA repair capacity.
\end{abstract}

Keywords: DNA double-strand breaks; Low-dose radiation; Normal tissue toxicity; DNA damage foci; 53BP1; Non-homologous endjoining

\section{Introduction}

Humans are regularly exposed to ionizing radiation, which can pose a health risk. Radioactive minerals in the earth and cosmic rays from outer space represent natural sources of radiation. Man-made sources of radiation also occur. Notably, nuclear power plant accidents have released radioactive isotopes into the environment, creating a potential health risk for nearby residents. A more common man-made exposure, however, is the radiation used during diagnostic and therapeutic medical procedures. Despite the ubiquity of exposure to low levels of radiation, the biological consequences and associated health effects remain unclear [1-3].

The present system of health risk evaluation and radiation protection is based on the linear-no-threshold assumption, in which the effects of high-dose radiation are extrapolated to the low-dose region of the exposure curve [4]. The primary sources of information on the health risks associated with radiation are epidemiological studies of exposed human populations, in particular the survivors of the Japanese atomic bombings. While the current system of radiation protection is based on population-averaged risk estimates, clinical evidence from diagnostic and therapeutic uses of ionizing radiation clearly shows that individuals respond differently to radiation exposure $[5,6]$. With regards to protection, it is very important to identify radiosensitive individuals and to understand the mechanisms involved. Given the ethical constraints of human research, the use and development of appropriate animal models are essential; in this way, we can perform mechanistic studies to improve our knowledge of living organisms' cellular responses to low quantities of ionizing radiation.

Exposure to ionizing radiation results in a wide range of DNA lesions. DNA double-strand breaks (DSBs) are considered the most biologically significant type of lesion, as the inability to repair DSBs leads to genomic instability and cell death. As a result, complex DNA damage responses have evolved to detect DSBs, promote the arrest of the cell cycle, and activate DNA repair pathways. In mammalian cells, the major pathway for DSB repair is non-homologous end joining (NHEJ). It operates throughout the cell cycle and does not require template DNA [7]. Two critical sensors and transducers of DSB signals are the kinases ataxia telangiectasia mutated (ATM) and DNAdependent protein kinase catalytic subunit (DNA-PKcs). They phosphorylate the histone variant $\mathrm{H} 2 \mathrm{AX}$ that flanks the regions of chromatin beside the break. Immunofluorescence can be used to visualize phosphorylated $\mathrm{H} 2 \mathrm{AX}(\gamma \mathrm{H} 2 \mathrm{AX})$ and other repair factors that co-localize to DSBs, such as 53BP1; they appear as discrete nuclear foci at the site of breaks [8].

In humans, several mutations are known to confer radio-sensitivity via compromised DSB repair. For example, severe combined immunodeficiency (SCID) is caused by mutations in DNA-PKcs, which lead to defective $\mathrm{V}(\mathrm{D}) \mathrm{J}$ recombination and extreme radiosensitivity $[9,10]$. Similarly, the neurodegenerative disorder, Ataxia telangiectasia (A-T) is caused by a mutation in ATM, which leads to increased radio-sensitivity, genomic instability, and predisposition to 
cancer [11]. Approximately $1 \%$ of the general population is a heterozygous carrier of an ATM mutation (ATM+/-). Although carriers do not exhibit the severe symptoms observed in ATM homozygotes (ATM-/-), they do exhibit increased cancer risk and are prone to serious radiotherapy side effects [12,13]. While severe DSB repair disorders, such as SCID and A-T, are extremely rare, it is likely that minor DSB repair deficiencies, due to more subtle, heterozygous mutations in DNA damage response genes, are more common [14]. The import of such mutations for health is not yet known.

In this study, we investigated the biological effects of fractionated low-dose radiation $(2,4,6,8$, and 10 weeks of daily 10 mGy radiation) in repair-proficient $(\mathrm{C} 57 \mathrm{BL} / 6, \mathrm{ATM}+/+)$, questionable repairproficient $(\mathrm{ATM}+/-)$ and deficient (ATM-/-, SCID) mouse strains. DSBs were monitored by measuring 53BP1 foci in the lung (bronchiolar and alveolar cells), heart (cardiomyocytes), and brain (cortical neurons). Our findings from this genetically heterogeneous, in vivo model help define the molecular and cellular processes underlying the response of healthy tissue to repetitive, low-dose radiation. Moreover, they may aid in the selection of appropriate animal models for studying long-term biological effects of low-dose exposure.

\section{Materials and Methods}

\section{Mouse strains and animal welfare}

The Jackson Laboratory derived the founder strain of the ATM (129S6/SvEvTac-Atmtm1Awb/J) mouse. The progeny obtained from crossing ATMtm1Awb/+ with ATMtm1Awb/+ mice were genotyped via PCR, using DNA from ear-punch tissue. Genotyping was carried out according to a protocol provided by The Jackson Laboratory. The PCR primers for the generic ATMtm1Awb allele (280-bp product) were $5^{\prime}$-CTT GGG TGG AGA GGC TAT TC-3' and 5'-AGG TGA GAT GAC AGG AGA TC-3'; the primers for the wild-type ATM allele (147-bp product) were 5 '-GCT GCC ATA CTT GAT CCA TG-3 ' and 5'-TCC GAA TTT GCA GGA GTT G-3'. PCR experiments were performed using the LightCycler $480^{\circ}$ Real-Time PCR System (Roche Applied Science ${ }^{\mathrm{rm}}$ ). In addition, C57BL/6 (C57BL/6NCrl) mice were obtained from Charles River Laboratories, Sulzfeld, Germany, and SCID (CB17/Icr-Prkdcscid/Rj) mice were obtained from Janvier, St. Berthevin Cedex, and France. All mice were housed in groups of 3 to 6 animals in IVC cages under standard laboratory conditions $\left(22^{\circ} \mathrm{C}\right.$ $\pm 2,55 \% \pm 10$ humidity, $12 \mathrm{~h}: 12 \mathrm{~h}$ light/dark cycle, ad libitum feeding conditions).

\section{Animal irradiation and tissue sampling}

For every irradiation session the mice were picked up from the animal care facility und brought to the Department of Radiation Oncology. For each experiment mice of one cage were placed in a plexiglass cylinder measuring $18 \mathrm{~cm}$ in diameter for whole-body irradiation with a linear accelerator (Artiste ${ }^{\mathrm{m} w}$, Siemens). Immediately after irradiation all animals were returned to the animal care facility. To improve the homogeneity of the photon beam, the cylinder was covered with $1.5-\mathrm{cm}$ thick tissue-equivalent plastic. The following conditions were employed: radiation field size: $30 \mathrm{~cm} \times 30 \mathrm{~cm}$, collimator angle $0^{\circ}$; gantry angle $0^{\circ}$; source surface distance (SSD): 208 $\mathrm{cm}$; beam energy: 6-MV photons; dose-rate: $2 \mathrm{~Gy} / \mathrm{min}$. Tomographybased, three-dimensional dose calculations were calculated using the Pinnacle ${ }^{\mathrm{TM}}$ planning system (Philips Radiation Oncology Systems, Fitchburg, WI). A thermo luminescent dosimeter was used to confirm reliable and uniform delivery of $10 \mathrm{mGy}$ protracted low-dose radiation. To evaluate induction and repair of DSBs after single-dose radiation, mice were examined $0.5,24$, and $72 \mathrm{~h}$ after exposure to 10 mGy. To evaluate the effects of daily low-dose radiation, mice were irradiated once every $24 \mathrm{~h}$ from Monday to Friday for 2, 4, 6, 8, or 10 weeks. 24 or $72 \mathrm{~h}$ after the final radiation treatment animals were intraperitoneally anesthesized with ketamin $(120 \mu \mathrm{g}$ per g body weight) and Rompun ${ }^{\star}$ (16 $\mu$ g per g body weight), killed by cervical dislocation before tissue collection. Tissues of three (C57BL/6: $14 \times 3$ animals; ATM+/+: $5 \times 3$ animals; ATM+/-: $5 \times 3$ animals) or two mice (ATM-/-: $5 \times 2$ animals; SCID: $5 \times 2$ animals, each with two independent replicas) per strain and per time-point were analyzed ( 92 mice in total). These studies were approved by the Medical Sciences Animal Care and Use Committee of the University of Saarland.

\section{Immunofluorescence}

Formalin-fixed tissues were embedded in paraffin and cut into 4$\mu \mathrm{m}$ thick sections. Wax was removed in xylene. Then, sections were rehydrated in decreasing concentrations of alcohol. Tissues were boiled in citrate buffer and pre-incubated with Roti ${ }^{\mathrm{TN}}-$ Immunoblock (Carl Roth, Karlruhe, Germany). Subsequently, sections were incubated with primary antibodies (anti-53BP1, Bethyl Laboratories, Montgomery, TX, USA) followed by incubation with an Alexa Fluor-488 secondary antibody (Invitrogen, Karlsruhe, Germany). Finally, sections were mounted in VECTAshield ${ }^{\text {ma }}$ with $4^{\prime}, 6-$ diamidino-2-phenylindole (DAPI; Vector Laboratories, Burlingame, CA, USA). A Nikon E600 epifluorescent microscope equipped with a charge-coupled device camera and acquisition software (Nikon, Düsseldorf, Germany) was used to capture fluorescence images. 53BP1 foci were quantified at objective magnification of $60 \times$ and $100 \times$ until at least 40 foci were registered per tissue sample. Accordingly, for each data point (comprising 3 or 4 tissue samples) between 400 and 4000 cells were screened for 53BP1 foci.

\section{Statistical Analysis}

Differences between the number of 53BP1 foci in irradiated and non-irradiated tissues were assessed using a one-side Mann-Whitney test carried out with OriginPro Software (Version 8.5, OriginLab Corporation, Northampton, MA). We considered p-values $\leq 0.05$ statistically significant.

\section{Results}

Tissues are organized communities of cells that work together to carry out specific functions in multicellular organisms. Healthy tissues are composed of cell populations with different rates of cell division and death. In this study, tissues composed primarily of non-dividing permanent cells were investigated to capture the accumulation of DNA damage after protracted low-dose radiation. In lung parenchyma, DNA damage was analyzed by counting 53BP1 foci in the columnar epithelial cells of bronchioles (bronchiolar cells) and in type-1 pneumocytes lining the alveoli (alveolar cells). In the heart, 53BP1 foci were enumerated in the nuclei of cardiomyocytes located in the muscular layer of the ventricles (striated muscular fiber). In the brain, cortical neurons in the gray matter along both hemispheres were analyzed for DNA damage accumulation. Immunofluorescence staining for 53BP1 revealed an increased number of radiation-induced foci in tissue exposed to fractionate low-dose radiation compared to non-irradiated lung, heart, and brain tissue (Figure 1). 
Citation: Schanz S, Flockerzi E, Schuberth K, Rübe CE (2014) Genetically-Defined DNA Repair Capacity Determines the Extent of DNA Damage Accumulation in Healthy Mouse Tissues after Very Low Doses of Ionizing Radiation. J Carcinog Mutagen 5: 196. doi: $10.4172 / 2157-2518.1000196$

Page 3 of 7

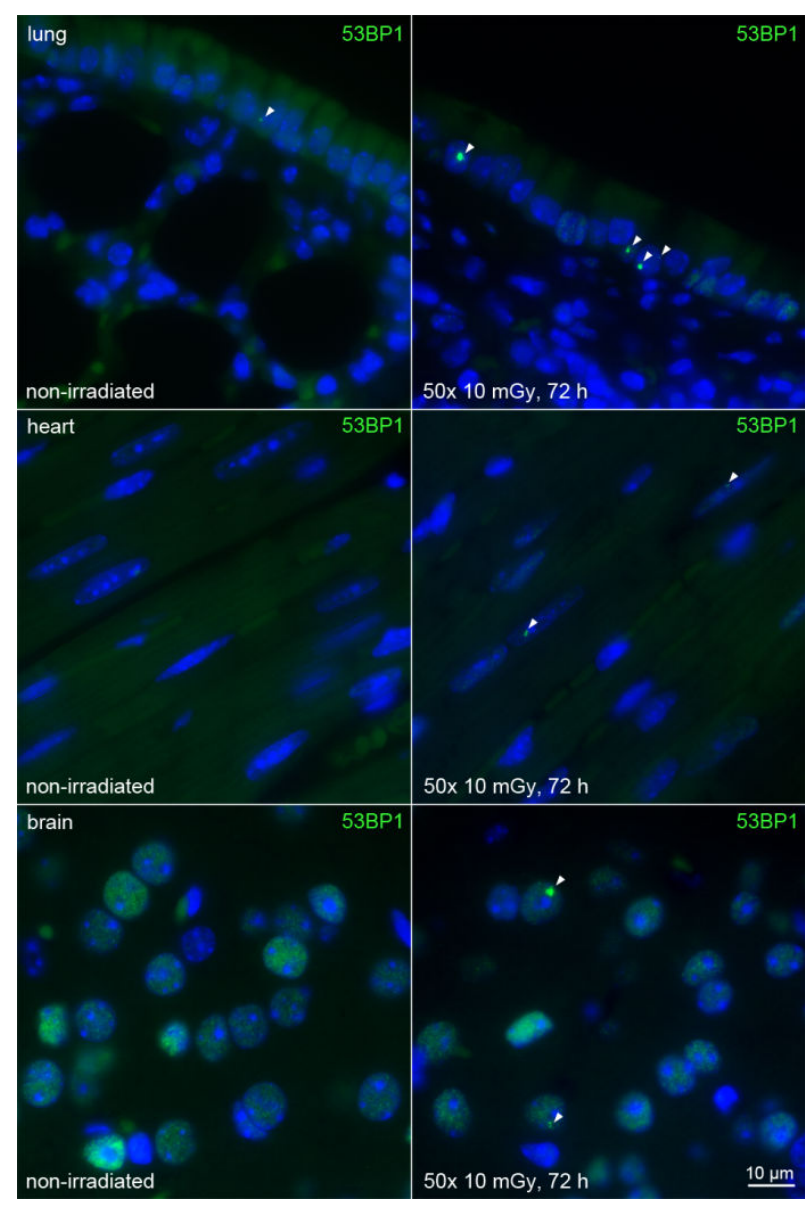

Figure 1: Radiation-induced foci in tissues from repair-proficient mice $(\mathrm{C} 57 \mathrm{BL} / 6)$ exposed to fractionated low-dose radiation. Immunofluorescent staining for 53BP1 (green) in DAPI-stained nuclei of bronchiolar and alveolar cells (lung), cardiomyocytes (heart), and cortical neurons (brain) analyzed $72 \mathrm{~h}$ after exposure to $50 \times 10 \mathrm{mGy}$, compared to non-irradiated control tissues. Original magnification, $600 \times$.

Background levels of 53BP1 must be consistently low to accurately quantify DSBs in response to low-dose radiation. Spontaneous formation of 53BP1 foci varied in the different cell populations of repair-proficient C57BL/6 mice. Bronchiolar cells had higher levels $(0.118 \pm 0.006 \mathrm{foci} /$ cell $)$ than alveolar cells $(0.048 \pm 0.003 \mathrm{foci} /$ cell $)$ (Figure 2). Cardiomyocytes exhibited moderate levels $(0.069 \pm 0.011$ foci/cell), and cortical neurons displayed the lowest levels of spontaneous foci $(0.031 \pm 0.003 \mathrm{foci} / \mathrm{cell})$. Tissue samples analyzed 0.5 $\mathrm{h}$ after single-dose radiation with $10 \mathrm{mGy}$ displayed significantly higher foci levels in all analyzed cell populations: $0.280 \pm 0.004$ foci/ cell in bronchiolar cells (8-fold increase), $0.199 \pm 0.008 \mathrm{foci} /$ cell in alveolar cells (12-fold increase), $0.192 \pm 0,018$ in cardiomyocytes (3fold increase), and $0.240 \pm 0.001$ in neurons (7-fold increase). Even 24 $\mathrm{h}$ after a single-dose exposure, foci levels were significantly elevated in bronchiolar cells $(0.238 \pm 0.024 \mathrm{foci} /$ cell $)$ and cardiomyocytes $(0.118 \pm$ $0.021 \mathrm{foci} / \mathrm{cell})$. However, $72 \mathrm{~h}$ after radiation exposure, foci in all cell populations had nearly returned to background levels (Figure 2). In subsequent fractionated low-dose experiments, residual 53BP1 foci were quantified 24 and $72 \mathrm{~h}$ after the last exposure for 2, 4, 6, 8 and 10 weeks of daily $10-\mathrm{mGy}$ radiation in repair-proficient mice. In all cell populations, the number of foci increased steadily with increasing cumulative radiation dose (Figure 2). The greatest number of foci was measured after 24 or $72 \mathrm{~h}$ exposure to $50 \times 10 \mathrm{mGy}$. Bronchiolar cells showed the greatest increase in foci, with a 2.5 -fold increase $(0.299 \pm$ $0.037 \mathrm{foci} /$ cell) relative to non-irradiated controls. The number of foci $24 \mathrm{~h}$ after exposure was consistently higher than at $72 \mathrm{~h}$, possibly reflecting ongoing repair. Collectively, our experimental data show that even for very low doses of ionizing radiation such as $10 \mathrm{mGy}$, there is no threshold for the induction of DNA damage.
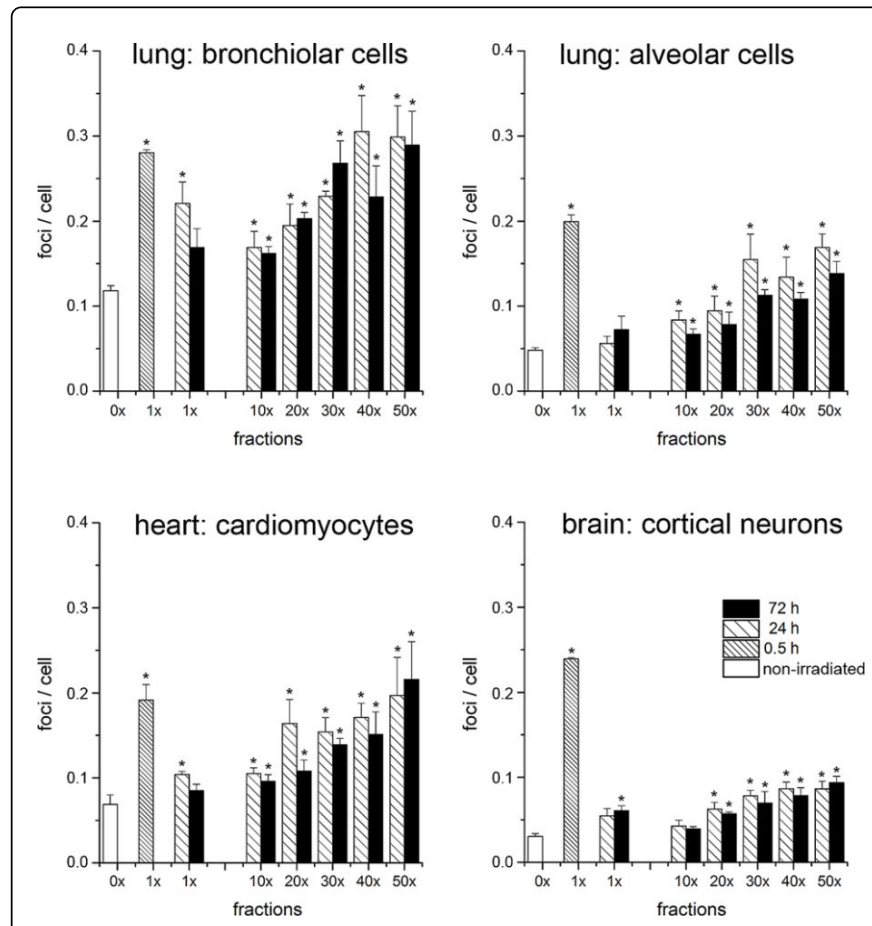

Figure 2: Quantification of 53BP1 foci in tissues from repairproficient mice $(\mathrm{C} 57 \mathrm{BL} / 6)$ exposed to fractionated low-dose radiation. The numbers of 53BP1 foci per nucleus were counted $0.5,24$, and $72 \mathrm{~h}$ after the first dose fraction $(1 \times)$ and 24 and $72 \mathrm{~h}$ after protracted low-dose radiation $(10 \times, 20 \times, 30 \times, 40 \times$, and $50 \times$ fractions over a time period of $2,4,6,8$, and 10 weeks) with 10 mGy. Data are presented as means from three different experiments \pm standard error. $\left({ }^{*}\right.$ denotes a statistically significant difference compared to non-irradiated control tissue).

We observed mouse strain-dependent differences in the levels of spontaneously occurring foci as well (Figure 3). In SCID mice, DNAPKcs mutations result in a truncated protein with impaired kinase activity that virtually inactivates the function of the DNA-PK complex. This pronounced DSB repair defect led to a significantly increased number of foci in non-irradiated SCID tissues, most pronounced in lung (bronchiolar cells: $0.442 \pm 0.030$ foci/cell; alveolar cells: $0.283 \pm$ $0.015 \mathrm{foci} / \mathrm{cell})$ and brain $(0.334 \pm 0.020 \mathrm{foci} / \mathrm{cell})$ tissue, though evident in the heart as well $(0.111 \pm 0.006 \mathrm{foci} /$ cell) (Figure 3$)$. In repair-deficient ATM-/- mice, significantly higher 53BP1 foci levels were observed primarily in the cortical neurons of the brain $(0.131$ \pm 0.024 foci/cell). Spontaneous $53 \mathrm{BP} 1$ foci in repair-deficient mice appeared to be extraordinarily large in size, with an average diameter 
Citation: Schanz S, Flockerzi E, Schuberth K, Rübe CE (2014) Genetically-Defined DNA Repair Capacity Determines the Extent of DNA Damage Accumulation in Healthy Mouse Tissues after Very Low Doses of Ionizing Radiation. J Carcinog Mutagen 5: 196. doi: $10.4172 / 2157-2518.1000196$

Page 4 of 7

of $1.45 \pm 0.054 \mu \mathrm{m}$ in SCID and $1.74 \pm 0.076 \mu \mathrm{m}$ in ATM-/- mice (compared to $0.80 \pm 0.040 \mu \mathrm{m}$ in repair-proficient C57BL/6 mice) (Figure 3). In addition, inter strain differences in 53BP1 background levels were observed among $\mathrm{C} 57 \mathrm{BL} / 6, \mathrm{ATM}+/+$, and $\mathrm{ATM}+/-$ mice, likely reflecting genetic variance in DSB repair capacity (Figure 3).
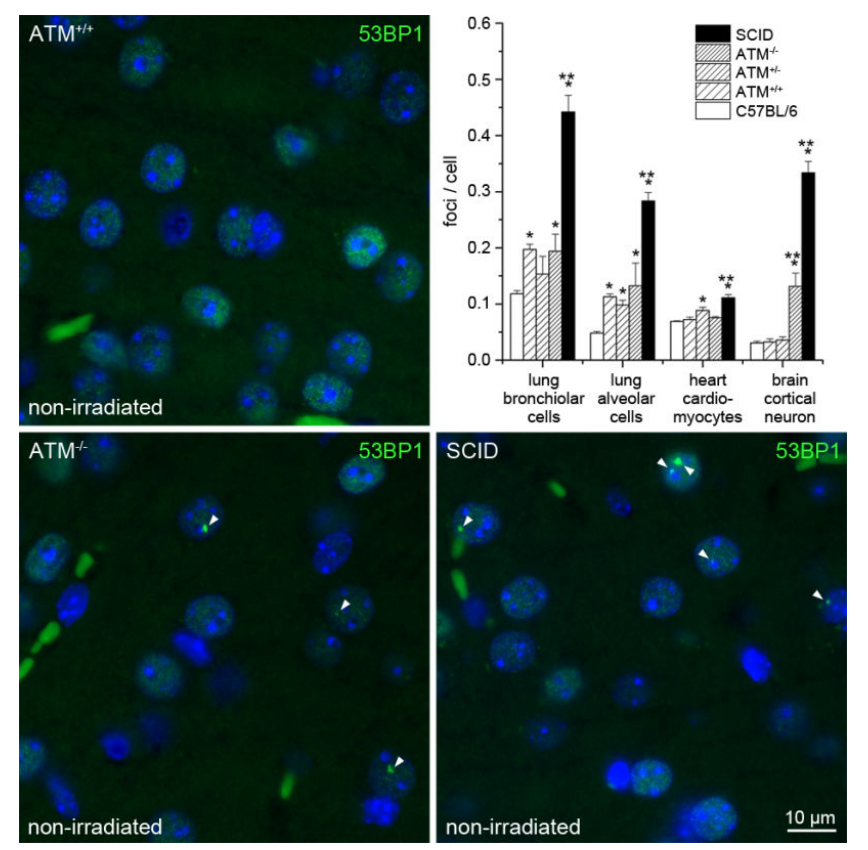

Figure 3: Increased numbers of 53BP1 foci in non-irradiated brain tissue from repair-deficient (ATM-/- and SCID) mice. Immunofluorescent staining for 53BP1 (green) demonstrated increased foci relative to repair-proficient $(\mathrm{ATM}+/+)$ mice. Original magnification, $600 \times$. Quantification of 53BP1 foci in nonirradiated tissues of $\mathrm{C} 57 \mathrm{BL} / 6, \mathrm{ATM}+/+$, ATM-/- and SCID mice. Data are presented as means from three different experiments \pm standard error. ( ${ }^{*}$ denotes a statistically significant difference compared to $\mathrm{C} 57 \mathrm{BL} / 6$ mice, ${ }^{* *}$ to $\mathrm{ATM}+/+$ mice) .

Next, we examined foci levels in tissues from ATM+/-, ATM-/-, and SCID mice exposed to protracted low-dose radiation (Figures 4 and 5). To rule out strain background effects, repair-proficient ATM $+/+$ mice were used as an additional control (Figures 4 and 5). Due to the increased radio-sensitivity of the ATM-/- and SCID mice, the fractionation scheme was limited to a maximum of 20 fractions at 10 mGy each. We observed clearly elevated foci levels in SCID mice relative to repair-proficient ATM+/+ mice (Figure 5). However, due to the high background levels in non-irradiated SCID tissues, especially in lung and brain, these radiation-induced elevations were not statistically significant after $10 \times 10 \mathrm{mGy}$ (Figure 5). We observed persistently high levels $24 \mathrm{~h}$ and $72 \mathrm{~h}$ post-irradiation with $20 \times 10$ $\mathrm{mGy}$, with values between $0.4-0.6$ foci/cell, significantly higher than baseline levels (Figure 5). The increased number of foci after multiple exposures reflected the pronounced DSB repair deficiency of SCID mice. In repair-deficient ATM-/- mice, multiple exposures to $10 \mathrm{mGy}$ significantly increased 53BP1 foci in all cell populations after $10 \times$ and $20 \times$ fractions, with maximal values between $0.2-0.5$ foci/cell (Figure 5). This persistent radiation-induced increase may be due to a defective ATM protein, leading to an accumulation of unrepaired DSBs. Healthy tissues from ATM+/- mice displayed similar numbers of foci as those of ATM+/+ mice after fractionated irradiation with 10 mGy (Figure 5). These results suggest that the repair capacity of ATM heterozygotes is sufficient to cope with DNA damage induced by lowdose radiation.

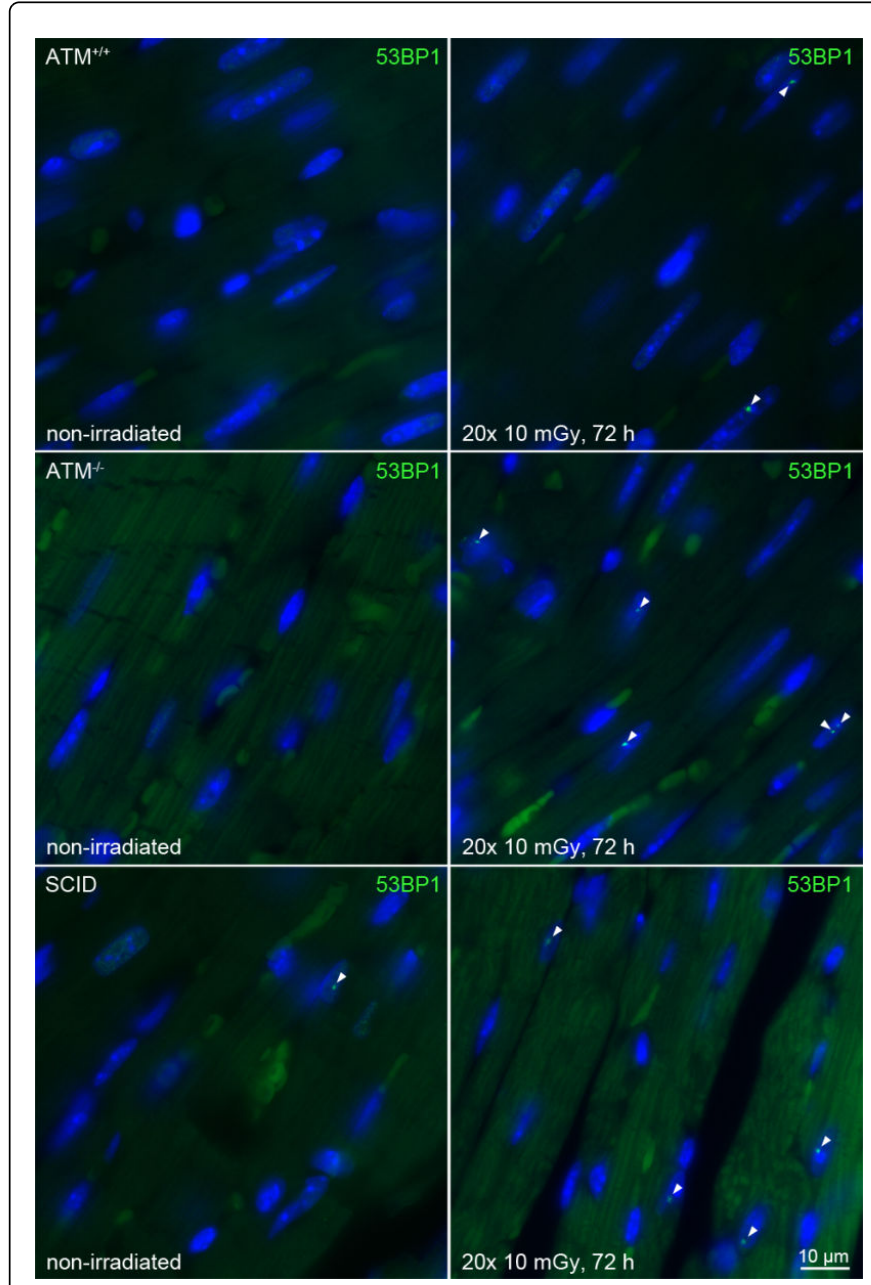

Figure 4: Increased numbers of 53BP1 foci in cardiomyocytes of ATM+/+, ATM-/- and SCID mice exposed to fractionated low-dose radiation. Tissue was analyzed $72 \mathrm{~h}$ after $20 \times 10 \mathrm{mGy}$ by immunofluorescent staining and compared to non-irradiated control. Original magnification $600 \times$. 
Citation: Schanz S, Flockerzi E, Schuberth K, Rübe CE (2014) Genetically-Defined DNA Repair Capacity Determines the Extent of DNA Damage Accumulation in Healthy Mouse Tissues after Very Low Doses of Ionizing Radiation. J Carcinog Mutagen 5: 196. doi: $10.4172 / 2157-2518.1000196$

Page 5 of 7
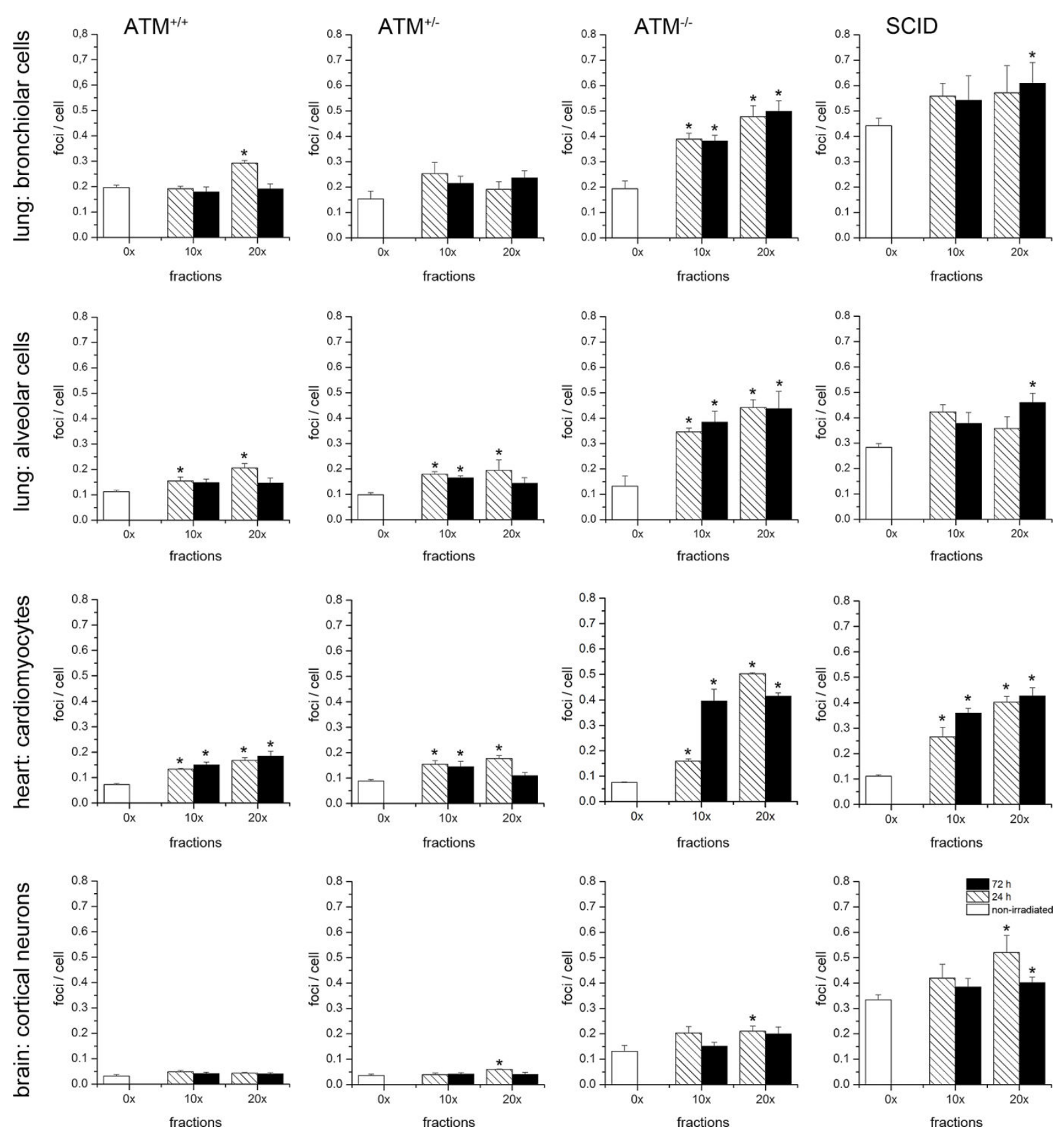

Figure 5: Quantification of 53BP1 foci in tissues from repair-proficient and -deficient mice exposed to fractionated low-dose radiation. The numbers of 53BP1 foci per nucleus in bronchiolar and alveolar cells (lung), cardiomyocytes (heart), and cortical neurons (brain) were counted 24 and $72 \mathrm{~h}$ after fractionated low-dose radiation $(10 \times$ and $20 \times$ fractions, $10 \mathrm{mGy}$ ) in repair-proficient (ATM $+/+$ ), questionable repairproficient $(\mathrm{ATM}+/-)$ and repair-deficient (ATM-/-, SCID) mouse strains. Data presented are means from four different samples \pm standard error. $\left({ }^{*}\right.$ denotes a significant difference compared to non-irradiated control tissue.)

\section{Discussion}

To understand the cellular mechanisms underlying the human response to low-dose ionizing radiation, the appropriate selection of an animal model is essential. After exposing mouse strains with different DNA repair capacity to repetitive low-dose radiation, we analyzed DNA damage in terminal, differentiated cell populations of complex tissues. Using 53BP1 as a marker of DSBs, we observed accumulation of DNA damage with increasing doses of fractionated irradiation. Levels of radiation-induced 53BP1 varied significantly between the different cell populations, suggesting varying levels of vulnerability to ionizing radiation. Moreover, genetic DNA repair capacity determined the extent of cumulative low-dose radiation damage, with no verifiable threshold-dose evident even in repairproficient organisms. The repair capacity of ATM heterozygotes was 
sufficient to cope with the DNA damage induced by repetitive lowdose radiation. Collectively, our findings suggest that even very low doses of DNA-damaging radiation may increase the health risk of individuals, particularly of those with compromised DNA repair capacity.

Research into the biological effects of low-dose radiation has been hindered by a lack of assays sensitive enough to measure the relevant molecular and cellular alterations. However, technical advances in visualizing and characterizing radiation-induced DNA damage in healthy tissues from living organisms provide powerful tools to uncover the molecular mechanisms underlying radiation-induced health effects and individual susceptibility. The DNA repair ability of a cell is vital to the integrity of its genome, and thus to the normal function of that organism. DSBs are the most lethal DNA lesion induced by ionizing radiation, and experimental animals lacking NHEJ proteins are dramatically radiosensitive. Human disorders resulting from defects in the NHEJ pathway, however, are extremely rare and normally have clinical features through which this radiosensitivity can be recognized [15]. It remains unknown whether more subtle changes in the same genes confer radio-sensitivity and whether heterozygosity affects the response to low-dose radiation. Here, we analyzed mouse strains with different genetic deficiencies in DSB repair to imitate the intrinsic variability within a human population. In repair-proficient and deficient strains, repeated exposure to low-dose radiation led to a substantial increase in DSB foci in all analyzed cell populations; the number of persistent foci was associated with the underlying repair defect and the cumulative radiation dose. These findings suggest that even in this low-dose range, there is a clear relationship between DSB repair capacity and radio-sensitivity in nondividing permanent cells, characterized by the absence of a definitive threshold dose even in repair-proficient organisms.

In healthy, non-irradiated tissues, reactive oxygen species generated during normal cellular metabolism represent the major DNAdamaging agents, though errors occurring during normal DNA replication provide additional contributions. Thus, even in the absence of radiation exposure, cells must constantly deal with a large number and wide range of mutagenic DNA lesions, and cancer risk estimates for radiation exposures must account for this background, or spontaneous, damage. In our study, non-irradiated tissues from mice with a defective NHEJ repair pathway revealed significantly increased foci numbers. These numbers were influenced by the underlying repair defect as well as by the cell population investigated. Our findings illustrate the important role of efficient DNA repair required for maintaining tissue homeostasis and underscore the requirement for constant response to DNA lesions to prevent tumorigenesis. However, the precise role of individual sensitivity to low-dose radiation exposure in cancer induction and susceptibility is poorly understood and requires further research.

The response of stem cells to radiation exposure is also an important determinant of overall tissue response, if recovery necessitates stem cell replication and/or differentiation of early progenitor cells. Moreover, stem cells of normal tissue may be important targets for radiation-induced carcinogenesis [16]. In a previous study, we showed that DNA damage response mechanisms are markedly different in stem cells, which are characterized by unique chromatin compositions $[17,18]$. In spermatogonial stem cells lacking compact heterochromatin, histone-associated signaling components of the DNA repair machinery are completely absent and radiationinduced DSBs are rejoined predominantly by DNA-PK-independent pathways, suggesting the existence of alternative repair mechanisms $[19,20]$.

In the present study, we analyzed terminally differentiated cell populations. Thus, the function and sensitivity of cell cycle checkpoints had no impact on DSB signaling response and radiosensitivity. We observed varying foci numbers among the different cell types, perhaps reflecting the cell-type specific efficiency of DSB rejoining. However, from previous electron microscopic analysis [21], we know that varying foci numbers may also reflect different subcellular foci distributions, due to cell type-specific chromatin organization [22]. Varying with cell type, the nuclear space in G0/G1phase cells is divided into different regions of low-density euchromatin and high-density heterochromatin. Thus, cell typespecific spatial variation in chromatin density may influence foci clustering, as visualized by immunofluorescence microscopy. The limited spatial resolution of immunofluorescence impedes discrimination between single foci in compact heterochromatin, thereby leading to underestimation of foci numbers. Our results show that foci can be used to examine DNA damage even after very low doses of radiation. However, due to the limited resolution of immunofluorescence microscopy, no constant proportionality between the number of DSBs and resulting foci could be assumed in our data.

The biological significance of persistent DNA damage foci with regard to radiation-induced health effects, however, is unclear. Our previous studies, exploring persistent 53BP1 foci based on transmission electron microscopy combined with immunogoldlabeling of repair factors, suggest that after exposure to low-dose radiation, nearly all DSBs are efficiently rejoined [22]. Recent studies have shown that the DNA damage response protein 53BP1 protects DNA ends from excessive resection in G1, and thereby favors repair by NHEJ as opposed to homologous recombination. In addition to DNA end-blocking activities, 53BP1 has been suggested to directly mediate long-range chromosomal interactions and DSB mobility that facilitates the juxtaposition of distal DNA ends [23]. Accordingly, persistent 53BP1 foci after radiation exposure may reflect lasting rearrangements of chromatin. Severe disruptions to the structure of chromatin, such as those associated with DSBs, facilitate damage-specific epigenetic responses [24]. This could potentially result in epigenetic regulatory defects with serious implications for gene expression [25-27].

In future studies we will characterize persistent, radiation-induced DNA damage foci and elucidate their biological significance for the fundamental processes of normal cellular functioning. One of the most important future challenges in low-dose radiation research is to establish to what extent ionizing radiation perturbs normal metabolism at the level of the cell, tissue, and organ, interfering with homeostatic equilibrium of the healthy organism.

\section{Acknowledgement:}

The research leading to these results has received funding from the European Atomic Energy Community's Seventh Framework Programme (FP7/2007-2011) under grant agreement $n^{\circ} 249689$ (Low Dose Research towards Multidisciplinary Integration).

\section{References}

1. Bonner WM (2003) Low-dose radiation: thresholds, bystander effects, and adaptive responses. Proc Natl Acad Sci U S A 100: 4973-4975. 
Citation: Schanz S, Flockerzi E, Schuberth K, Rübe CE (2014) Genetically-Defined DNA Repair Capacity Determines the Extent of DNA Damage Accumulation in Healthy Mouse Tissues after Very Low Doses of Ionizing Radiation. J Carcinog Mutagen 5: 196. doi: $10.4172 / 2157-2518.1000196$

Page 7 of 7

2. Brenner DJ, Doll R, Goodhead DT, Hall EJ, Land CE, et al. (2003) Cancer risks attributable to low doses of ionizing radiation: assessing what we really know. Proc Natl Acad Sci U S A 100: 13761-13766.

3. Hall EJ, Brenner DJ (2008) Cancer risks from diagnostic radiology. Br J Radiol 81: 362-378.

4. Puskin JS (2009) Perspective on the use of LNT for radiation protection and risk assessment by the U.S. Environmental Protection Agency. Dose Response 7: 284-291.

5. Rübe CE, Fricke A, Schneider R, Simon K, Kühne M, et al. (2010) DNA repair alterations in children with pediatric malignancies: novel opportunities to identify patients at risk for high-grade toxicities. Int J Radiat Oncol Biol Phys 78: 359-369.

6. Schuler N, Palm J, Kaiser M, Betten D, Furtwängler R, et al. (2014) DNAdamage foci to detect and characterize DNA repair alterations in children treated for pediatric malignancies. PLoS One 9: e91319.

7. Chapman JR, Taylor MR, Boulton SJ (2012) Playing the end game: DNA double-strand break repair pathway choice. Mol Cell 47: 497-510.

8. Vignard J, Mirey G, Salles B (2013) Ionizing-radiation induced DNA double-strand breaks: a direct and indirect lighting up. Radiother Oncol 108: 362-369.

9. van der Burg M, Ijspeert H, Verkaik NS, Turul T, Wiegant WW, et al. (2009) A DNA-PKcs mutation in a radiosensitive T-B- SCID patient inhibits Artemis activation and nonhomologous end-joining. J Clin Invest 119: 91-98.

10. Woodbine L, Neal JA, Sasi NK, Shimada M, Deem K, et al. (2013) PRKDC mutations in a SCID patient with profound neurological abnormalities. J Clin Invest 123: 2969-2980.

11. Lavin MF (2008) Ataxia-telangiectasia: from a rare disorder to a paradigm for cell signalling and cancer. Nat Rev Mol Cell Biol 9: 759-769.

12. Barlow C, Eckhaus MA, Schäffer AA, Wynshaw-Boris A (1999) Atm haploinsufficiency results in increased sensitivity to sublethal doses of ionizing radiation in mice. Nat Genet 21: 359-360.

13. Olsen JH, Hahnemann JM, Børresen-Dale AL, Brøndum-Nielsen K, Hammarström L, et al. (2001) Cancer in patients with ataxiatelangiectasia and in their relatives in the nordic countries. J Natl Cancer Inst 93: 121-127.

14. Garber JE, Offit K (2005) Hereditary cancer predisposition syndromes. J Clin Oncol 23: 276-292.

15. Woodbine L, Gennery AR, Jeggo PA (2014) The clinical impact of deficiency in DNA non-homologous end-joining. DNA Repair (Amst) 16C: 84-96.
16. Manda K, Kavanagh JN, Buttler D, Prise KM, Hildebrandt G (2014) Low dose effects of ionizing radiation on normal tissue stem cells. Mutat Res Rev Mutat Res .

17. Schanz S, Schuler N, Lorat Y, Fan L, Kaestner L, et al. (2012) Accumulation of DNA damage in complex normal tissues after protracted low-dose radiation. DNA Repair (Amst) 11: 823-832.

18. Schuler N, Rübe CE (2013) Accumulation of DNA damage-induced chromatin alterations in tissue-specific stem cells: the driving force of aging? PLoS One 8: e63932.

19. Rübe CE, Zhang S, Miebach N, Fricke A, Rübe C (2011) Protecting the heritable genome: DNA damage response mechanisms in spermatogonial stem cells. DNA Repair (Amst) 10: 159-168.

20. Moscariello M, Iliakis G (2013) Effects of chromatin decondensation on alternative NHEJ. DNA Repair (Amst) 12: 972-981.

21. Rube CE, Lorat Y, Schuler N, Schanz S, Wennemuth G, et al. (2011) DNA repair in the context of chromatin: new molecular insights by the nanoscale detection of DNA repair complexes using transmission electron microscopy. DNA Repair (Amst) 10: 427-437.

22. Lorat Y, Schanz S, Schuler N, Wennemuth G, Rube C, et al. (2012) Beyond repair foci: DNA double-strand break repair in euchromatic and heterochromatic compartments analyzed by transmission electron microscopy. PLoS One 7: e38165.

23. Callen E, Di Virgilio M, Kruhlak MJ, Nieto-Soler M, Wong N, et al (2013) 53BP1 mediates productive and mutagenic DNA repair through distinct phosphoprotein interactions. Cell 153: 1266-1280.

24. Aypar U, Morgan WF, Baulch JE (2011) Radiation-induced epigenetic alterations after low and high LET irradiations. Mutat Res 707: 24-33.

25. Antwih DA, Gabbara KM, Lancaster WD, Ruden DM, Zielske SP (2013) Radiation-induced epigenetic DNA methylation modification of radiation-response pathways. Epigenetics 8: 839-848.

26. Forrester HB, Li J, Hovan D, Ivashkevich AN, Sprung CN (2012) DNA repair genes: alternative transcription and gene expression at the exon level in response to the DNA damaging agent, ionizing radiation. PLoS One 7: e53358.

27. O'Hagan HM (2014) Chromatin modifications during repair of environmental exposure-induced DNA damage: a potential mechanism for stable epigenetic alterations. Environ Mol Mutagen 55: 278-291. 